\title{
Subgingival periodontal pathogens associated with chronic periodontitis in Yemenis
}

\author{
Nezar N Al-hebshi ${ }^{1,2^{*}}$, Hussein M Shuga-Aldin ${ }^{3}$, Ali K Al-Sharabi ${ }^{3}$ and Ibrahim Ghandour ${ }^{4}$
}

\begin{abstract}
Background: Subgingival microbial profile associated with periodontitis has been reported to significantly differ by geographical location. The purpose of this study was to assess the association between a panel of putative periodontal bacterial pathogens and chronic periodontitis among Yemenis.

Methods: Subgingival DNA samples were obtained from diseased and healthy sites of 20 non-smoking, moderate to severe chronic periodontitis subjects. Absolute counts (bacterial DNA copies per sample) and relative counts (\% total bacteria) of seven periopathogenic species/genera representative of the red and orange complexes were determined using Taqman q-PCR assays.

Results: The q-PCR assays showed excellent linearity $\left(R^{2}>0.99\right)$ and a sensitivity of 100 copies/sample. The detection rate was $100 \%$ for all tested species/genera except for $P$. gingivalis and $A$. actinomycetemcomitans that were detected at $97.5 \%$ and $67.5 \%$, respectively. The median log absolute counts were in the range of 2.41-6.53 copies per sample while median relative counts were in the range of $0.001-0.77 \%$, both being highest for fusobacteria and lowest for A. actinomycetemcomitans. Significant interspecies correlations were observed. Adjusting for multiple comparisons (P $\leq 0.0063)$, only T. forsythia, T. denticola and P. micra maintained significant association with periodontal destruction. The latter species, however, showed the strongest association and was found in higher proportions at the periodontitis sites across all subjects (3.39 median fold increase). No significant differences were observed for $P$. gingivalis.
\end{abstract}

Conclusions: P. micra rather than P. gingivalis appears as a keystone pathogen in this Yemeni Sample. However, these findings need to be validated in a larger-scale study before they can be claimed to represent ethnic variations in pathogens' association with periodontitis.

Keywords: Microbiology, Pathogens, Real-time PCR, Parvimonas micra, Periodontitis

\section{Background}

Periodontitis represents a range of clinical entities that are characterized by immunological destruction of the tooth supporting structures in response to chronic challenge by specific bacteria in subgingival biofilm [1]. The last three decades or so witnessed an explosion in our understanding of the microbiology of periodontitis. Earlier studies employing cultivation-based techniques recovered about 250 bacterial species (at $>1 \%$ relative abundance) from plaque samples [2]. With the extensive application of molecular techniques over the last decade,

\footnotetext{
* Correspondence: nazhebshi@yahoo.com

'Department of Preventive Dentistry, Faculty of Dentistry, Jazan University,

P.O. box: 114, Jazan, Saudi Arabia

${ }^{2}$ Molecular Research Laboratory, Faculty of Medical Sciences, University of

Science and Technology, Sana'a, Yemen

Full list of author information is available at the end of the article
}

double this number of novel species has been identified [3-5] bringing the richness of the subgingival microbiota to more than 700 bacterial species, about $50 \%$ of which are uncultivable.

While the majority of subgingival microbiota is considered commensal, several species have been implicated as periodontal pathogens. Porphyromonas gingivalis, Tannerella forsythia, and Treponema denticola (the so called red complex) have so far shown the strongest association with chronic periodontitis [6]. Other putative pathogens include Fusobacterium spp., Prevotella spp., Campylobacter rectus, Eubacterium nodatom, and Parvimonas micra (previously Peptostreptococcus micros) [6]. Uncultivable phylotypes such as Synergistetes, TM7 and Treponema taxa are also believed to play a pathogenic role in chronic periodontitis $[3,4,7]$. In fact, it is believed that periodontal 
destruction is triggered by a bacterial consortium rather than a single pathogen [1].

A number of molecular techniques have been employed for detection and quantification of periodontal pathogens in plaque samples including DNA-DNA hybridization, conventional and real time PCR, and 16S rRNA clone sequencing [8]. Of these, real-time PCR is the most sensitive allowing detection of as low as 1.6 cells per reaction $[9,10]$. It also makes it possible to normalize target DNA counts to total bacterial counts in the sample (relative quantification), thus adjusting for variations in sampling and making comparisons between samples more reliable $[11,12]$. Surprisingly, real-time PCR has not been as widely used in the study of microbiology of periodontitis as may be expected.

Subgingival microbial profile associated with periodontitis have been reported to significantly differ by geographical location independent of other factors known to modify subgingival microbial composition $[8,13]$. It becomes prudent, therefore, that obtaining more information about the global distribution of periodontal pathogens and patterns of their association with disease can improve our understanding of the differences in the role they play in periodontitis in different populations. In the absence of data on this from the Middle East, the objective of the current study was to assess the association of seven putative periodontal pathogens with chronic periodontitis in a Yemeni population using quantitative PCR assays.

\section{Methods}

\section{Study subjects and clinical examination}

Twenty subjects, $30-50$ years old, with moderate to severe chronic periodontitis (having at least 1 site per quadrant with pocket depth $\geq 5 \mathrm{~mm}$ and attachment loss $>3 \mathrm{~mm}$ ), were recruited from among patients attending dental clinics at Al-thawra hospital, Sana'a, Yemen. Subjects presenting with less than 20 teeth or diagnosed with aggressive periodontitis (those with typical first molar/central incisor presentation) were excluded. Other exclusion criteria included history of smoking, periodontal treatment or antibiotic/oral antiseptic use in the previous 6 months, pregnancy or breast feeding, and any systemic disease or medication intake known to modify periodontal inflammation.

The community periodontal index [14] was used to screen periodontal status by a single, well-trained and precaliberated examiner (Shuga-aldin HM). In eligible subjects, pocket depth (PD) for the deepest pocket in each quadrant in millimeters was established using a Williams probe. The plaque index [15], was measured on the labial/buccal and lingual/palatal surfaces of index teeth. The clinical characteristics of the study group are shown in Table 1.
The study was carried in compliance with the Helsinki declaration. It was approved by the Medical and Health Studies Board, Graduate College, Khartoum University. Informed consent was obtained from all subjects.

\section{Sampling and DNA extraction}

For each subject, one pooled subgingival sample from the deepest pocket in each quadrant $(P D \geq 5 \mathrm{~mm})$ and another from 4 healthy sites $(\mathrm{PD} \leq 3 \mathrm{~mm}$; no attachment loss) were obtained, using sterile paper points. Supragingival plaque was removed prior to sampling using sterile cotton pellets. The samples (40 in total) were stored in low EDTA TE buffer (Invitrogen, USA) at $-80^{\circ} \mathrm{C}$ until processing.

At the time of DNA extraction, samples were centrifuged at $15,000 \mathrm{~g}$ for 1 minute and the pellet was resuspended in $180 \mu \mathrm{l}$ lysozyme digestion buffer $(25 \mathrm{mM}$ Tris- $\mathrm{HCl}$, $\mathrm{pH}$ 8.0, $2.5 \mathrm{mM}$ EDTA, 1\% Triton X-100) containing $20 \mathrm{mg} / \mathrm{ml}$ lysozyme, and incubated at $37^{\circ} \mathrm{C}$ overnight. The digest was then subject to DNA extraction using the Purelink Genomic DNA extraction kit (Invitrogen, USA); DNA was eluted in $100 \mu \mathrm{l}$ of the supplied buffer and stored at $4{ }^{\circ} \mathrm{C}$ for subsequent analysis.

\section{Quantitative PCR assays}

Total bacteria, Fusobacterium spp., Prevotella spp., Aggregatibacter actinomycetemcomitans (previously Actinobacillus actinomycetemcomitans), Parvimonas micra (previously Micromonas micra or Peptostreptococcus micros), Porphyromonas gingivalis, Tannerella forsythia, and Treponema denticola were detected and quantified in the DNA extracts using Taqman real-time PCR technology [16]. Sequences of probes and primers used in the study are shown in Table 2 . They were supplied by Primerdesign, UK, as optimized and ready to use kits which also included plasmid-based positive control (amplicon sequence inserted). The latter was serially diluted to construct standard curves for absolute quantification of the test species, and to assess efficiency, linearity and sensitivity of the assays.

To check for specificity, primers' sequences were first blasted against eubacterial sequences database at the National Center for Biotechnology Information (NCBI; http://www.ncbi.nlm.nih.gov/tools/primer-blast/index.cgi? LINK_LOC=BlastHome). Then, each set was tested in a SYBR Green real-time PCR assay against a pooled subgingival DNA sample from 5 periodontitis patients, followed by disassociation curve analysis. A primer set was judged as being specific if it resulted in a single disassociation peak that is identical to the positive standard peak.

Each reaction comprised of $10 \mu \mathrm{l}$ mastermix with ROX (Primerdesign, UK), $1 \mu \mathrm{l}$ primers/probe mix, $5 \mu \mathrm{l}$ template DNA (or positive standard), and $4 \mu \mathrm{l}$ PCR-grade water; all 
Table 1 Clinical characteristics of the study group

Gender (M/F \%) $60 / 40$

Age, median (interquartile range)

$40(30-45)$

Plaque index, median (interquartile range)

$1.5(1.25-1.65)$

Pocket depth at sampled sites, median (interquartile range) 5.5 (5.00-6.75)

runs were carried out on an ABI 7000 real-time PCR platform (Applied Biosystems, USA) using the following program: initial enzyme activation at $95^{\circ} \mathrm{C}$ for $10 \mathrm{~min}$ followed by 40 cycles of denaturation at $95^{\circ} \mathrm{C}$ for $15 \mathrm{sec}-$ onds and annealing/extension at $60^{\circ} \mathrm{C}$ for $1 \mathrm{~min}$. Data were acquired through the FAM channel.

Absolute counts, in copies/reaction, were calculated using the standard curves; these were then converted into copies/sample by multiplying by 20 (since $5 \mu \mathrm{l}$ of the extract was included in the reaction). Relative counts of the test species/genera were then calculated as \% total bacteria.

\section{Statistical analysis}

Examining clinical and microbiological data with the Kolmogorov-Smirnov statistic revealed non-normal distribution. Consequently, they were summarized as medians and interquartile ranges (IQR). Significance of differences between healthy and periodontitis sites in terms of absolute (log-transformed) and relative counts were sought using the Wilcoxon-signed rank test. Bonferroni's correction for multiple comparison was applied so an adjusted p-value of 0.0063 was used to describe significant difference. All tests were performed using SPSS 17 (SPSS Inc., Chicago, IL, USA).

\section{Results}

\section{The quantitative PCR assays}

All eight real-time PCR assays showed excellent linearity $\left(R^{2} \geq 0.99\right)$ over a dynamic range of $5-10^{6}$ copies/reaction (Figure 1), achieving a sensitivity of 100 copies/sample (given DNA extraction was $100 \%$ efficient). All primer sets produced single disassociation peaks in the SYBR Green assays that corresponded to the standard peaks (Figure 2).

\section{General microbiological findings}

All tested species/genera were detected in 100\% of the samples except $P$. gingivalis and $A$. actinomycetemcomitans,

Table 2 Sequences of primers and probes used in the quantitative PCR assays

\begin{tabular}{|c|c|c|c|c|}
\hline Test species & Sequences 5'-3' & Target gene & Product size & Ref \\
\hline \multirow[t]{3}{*}{ A. actinomycetemcomitans } & F-primer: GGRAGAATGGATGGCGATAT & & & \\
\hline & R-primer: ATCAGAATGAACATAACCTATACCA & hgpA & $81 \mathrm{bp}$ & This study \\
\hline & Probe: FAM- ATGAACGCAATTCAGCCCAGA ACTG-BHQ & & & \\
\hline \multirow[t]{3}{*}{ P. micra } & F-primer: TGAGCAACCTACCTTACACAG & & & \\
\hline & R-primer: GCCCTTCTTACACCGATAAATC & $16 \mathrm{~S}$ rRNA & $112 \mathrm{bp}$ & {$[17]$} \\
\hline & Probe: FAM- ACCGCATGAGACCACAGAA TCGCA-BHQ & & & \\
\hline \multirow[t]{3}{*}{ P. gingivalis } & F-primer: ACGAATCAAAGGTGGCTAAGTT & & & \\
\hline & R-primer: TTAGTCGCATTTTCGGCTGAT & $\operatorname{fim} A$ & $85 \mathrm{bp}$ & [17] \\
\hline & Probe: FAM- CCTGCTGTTCTCCATTATAAAC CATTACGG -BHQ & & & \\
\hline \multirow[t]{3}{*}{ T. forsythia } & F-primer: GATAGGCTTAACACATGCAAGTC & & & \\
\hline & R-primer: GTTGCGGGCAGGTTACATAC & $16 \mathrm{~S}$ rRNA & $99 \mathrm{bp}$ & [17] \\
\hline & Probe: FAM- TTACTCACCCGTGCGCCGGTCG-BHQ & & & \\
\hline \multirow[t]{3}{*}{ T. denticola } & F-primer: GGGCGGCTTGAAATAATRATG & & & \\
\hline & R-primer: CTCCCTTACCGTTCGACTTG & $16 \mathrm{~S}$ rRNA & $92 \mathrm{bp}$ & [17] \\
\hline & Probe: FAM- CAGCGTTCGTTCTGAGCCA GGATCA-BHQ & & & \\
\hline \multirow[t]{3}{*}{ Total bacteria } & F-primer: AAACTCAAAGGAATTGACGGGG & & & \\
\hline & R-primer: TTGCGCTCGTTGCGGGACT & $16 \mathrm{~S}$ rRNA & $205 \mathrm{bp}$ & [17] \\
\hline & Probe: FAM-CTGTCGTCAGCTCGTGTCGTGA-BHQ & & & \\
\hline \multirow[t]{3}{*}{ Fusobacterium spp.* } & F-primer: CGCAGAAGGTGAAAGTCCTGTAT & & & \\
\hline & R-primer: TGGTCCTCACTGATTCACACAGA & $23 \mathrm{~S}$ r RNA & $101 \mathrm{bp}$ & [18] \\
\hline & Probe: FAM- CTTTGCTCCCAAGTAACATG GAACACGA-BHQ & & & \\
\hline \multirow[t]{3}{*}{ Prevotella spp.* } & F-primer: ACCAGCCAAGTAGCGTGCA & & & \\
\hline & R-primer: TGGACCTTCCGTATTACCGC & $16 \mathrm{~S}$ rRNA & $153 \mathrm{bp}$ & [19] \\
\hline & Probe: FAM- AATAAGGACCGGCTAATTCC GTGCCAG -BHQ & & & \\
\hline
\end{tabular}




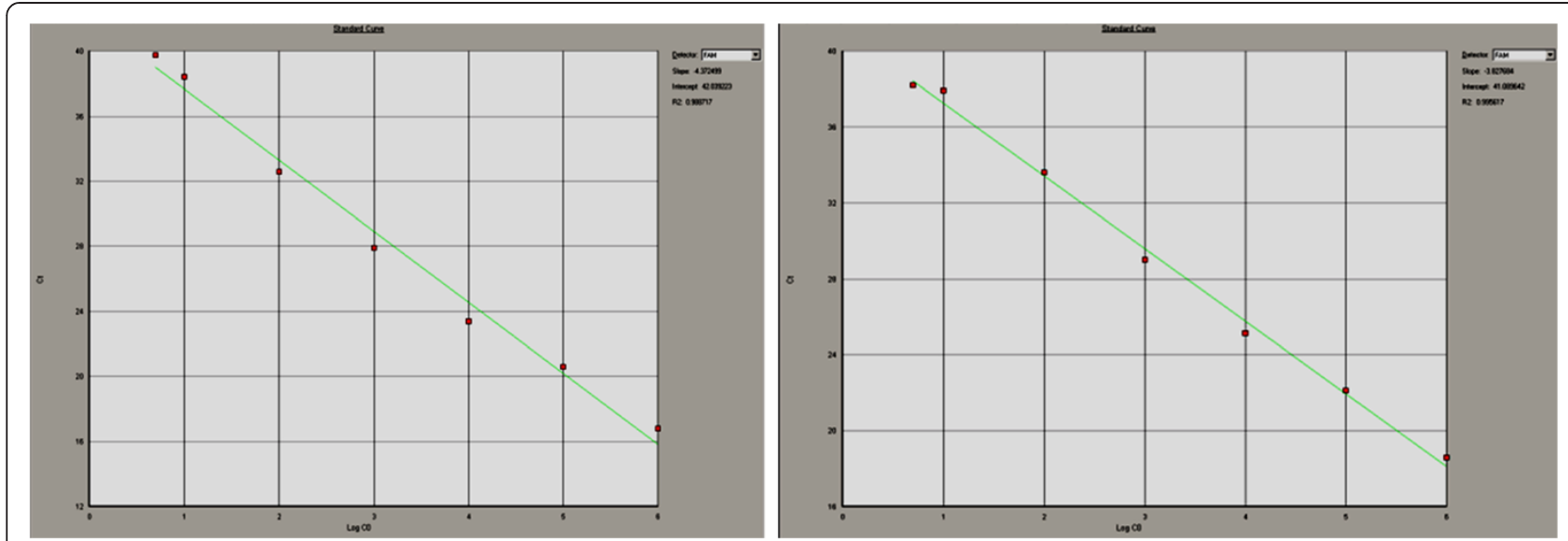

Figure 1 Screen shots of ABI 7000 SDS software's output showing standard curves for two of the primers/probe sets used in this study ( $T$. denticola to the left and $A$.actinomycetemcomitans to the right). Serial dilutions of plasmid-based positive control were prepared with final concentrations of $5-10^{6}$ copies/reaction. Assays were run as described in the text. The curves were obtained by plotting log DNA copies count against threshold cycle values $\left(C_{t}\right)$.

for which the detection rates were $97.5 \%$ and $67.5 \%$, respectively. Overall absolute and relative counts data are presented in Figure 3. The median log absolute count was 8.69 for total bacteria and in the range of 2.41-6.53 for individual species, being highest for fusobacteria and lowest for $A$. actinomycetemcomitans. Median relative counts (\% total bacteria) were in the range of 0.001-0.77\%, again being highest for fusobacteria and lowest for A. actinomycetemcomitans. Total pathogens (sum of all 7 species/genera) constituted 2.1\% (IQR $1.36-3.87 \%)$. No species was detected at higher than $1 \%$ in $75 \%$ of the samples. A actinomycetemcomitans, $P$. gingivalis and $P$. micra were never detected at more than $1 \%$ while fusobacteria, prevotellae, $T$. denticola and T. forsythia reached as far as $5.3 \%, 2.1 \%, 2.3 \%$ and $4.1 \%$, respectively, in outliers.

Non-parametric analysis (Spearman correlation) revealed a number of significant inter-species correlations. Based on log counts, and after performing Bonferroni correction for multiple comparisons $(\mathrm{P} \leq 0.002)$, fusobacteria showed significant correlation with prevotellae and P. micra $(\mathrm{r}=0.71$ and 0.53 , respectively), while $T$. denticola significantly correlated with $T$. forsythia $(\mathrm{r}=0.72)$. Almost the same patterns of associations were found based on proportions, but the correlation between fusobacteria and P. micra disappeared. Taking a less conservative level of significance

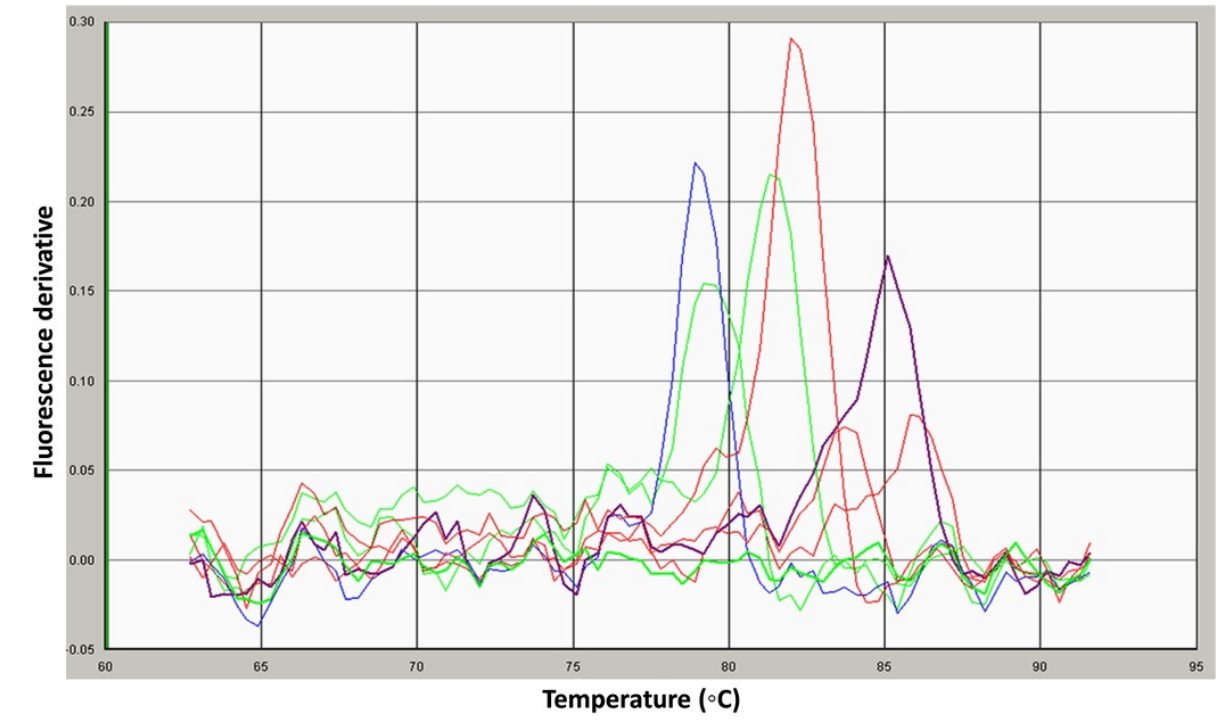

Figure 2 Disassociation curve analysis of amplicons produced by testing each of the primer pairs used in the study against pooled subgingival DNA sample from five periodontitis subjects using a Syber Green PCR assay. Each pair resulted in only one peak indicating specific amplification. 

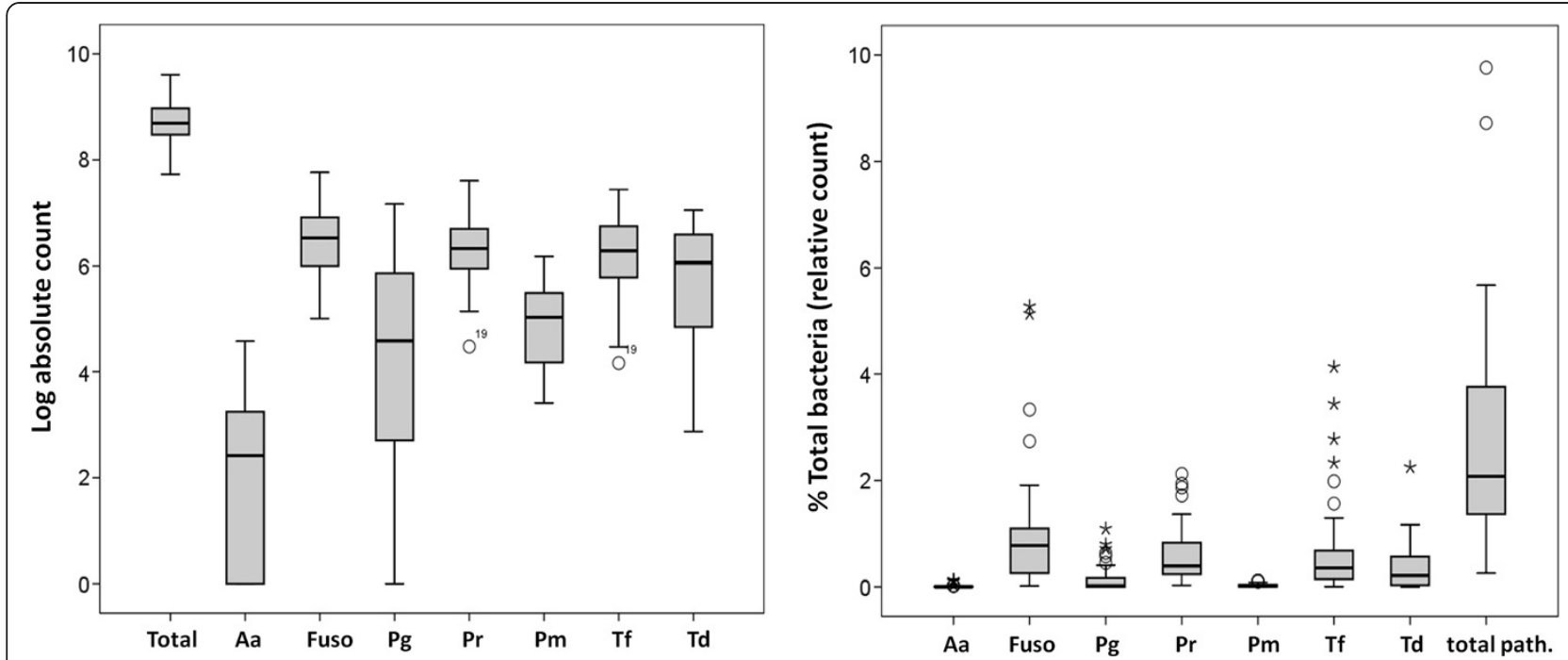

Figure 3 Box plots showing the median and interquartile range (IQR) of overall absolute (left) and relative (right) counts of test species/ genera in subgingival biofilm samples. The error bars represent data within 1.5 IQR above Q3 (third quartile) and below Q1 (first quartile). Circles and stars are outliers. Aa; A. actinomycetemcomitans; Fuso: fusobacteria; Pg: P. gingivalis; Pr: prevotellae; Pm: Parvimonas micra; Tf: T. forsythia; Td: T. denticola.

( $\mathrm{P} \leq 0.01), T$. forsythia was also found to positively correlate with fusobacteria and prevotellae.

\section{Periodontitis vs. healthy sites}

The absolute log counts of the test species/genera in the subgingival plaque samples from the healthy and periodontitis sites are presented in Table 3. More total bacterial DNA was recovered from the periodontitis sites than from the healthy sites although this did not withstand adjustment for multiple comparisons. All test species/genera, with the exception of $A$. actinomycetemcomitans and Fusobacterium spp., were also present at higher levels at sites with periodontal destruction; however, only P. micra and $T$. forsythia maintained significant difference after correction for multiple comparisons ( $\mathrm{P} \leq 0.0063)$.

Using relative counts (proportions), all test species, except $A$. actinomycetemcomitans, were present at higher proportions in the periodontitis sites compared to the healthy sites (Table 4). However, differences were only significant for $P$. micra and T. denticola $(\mathrm{P} \leq 0.0063)$ that showed 3.39 and 1.33 median fold increase, respectively, in sites with periodontal destruction compared to sites with no destruction. P. micra was present at higher relative counts in periodontitis compared to healthy sites across all subjects.

\section{Discussion}

Studies from the Middle East are limited to those that assessed the effect of traditional oral hygiene such as miswak, or certain habits, like qat chewing, on levels of periodontal pathogens [17,20,21]; no studies have so far assessed which periodontal pathogens are particularly associated with periodontitis in an Arab population. The study compared counts of 7 putative pathogens between healthy and diseased sites in patients with moderatesevere chronic periodontitis, using real-time PCR. Despite the advantage of this techniques (sensitivity and possibility of relative quantification), a limited number of studies used it in the study of microbiology of periodontitis and even fewer used relative quantification for making comparisons between health and disease. Healthy sites within the same subjects rather than healthy individuals were used as controls to avoid the effects of inter-individual variations in factors other than microbial composition. Nevertheless, including healthy subjects as additional controls would have allowed for more comparisons and a broader view of differences in microbial composition between periodontal health and disease. So this could be considered as one limitation of the current study. Another limitation is that bleeding on probing was not recorded although it has been previously shown to be an important clinical variable. In addition, while the most important pathogens were tested, the panel could have included more species particularly the newer pathogens such as Filifactor alocis, oral synergistetes and TM7.

Absolute counts were reported in DNA copies rather than cell numbers because the target gene copy number per genome, particularly $16 \mathrm{~S}$ rRNA gene, varies from one species to another and is not known for some of them. This implies that actual bacterial counts for some of the tested species/genera are less than the counts reported in the study. It, however, does not influence on validity of comparisons between sites or patients. Theoretically, as little as one gene copy per reaction can be 
Table 3 Median (interquartile range) log counts of the test species/genera in subgingival plaque from the healthy and periodontitis sites

\begin{tabular}{lccc}
\hline Species & Healthy sites $(\mathbf{n}=\mathbf{2 0})$ & Periodontitis sites $(\mathbf{n}=\mathbf{2 0})$ & \multicolumn{1}{c}{} \\
\hline Total bacteria & $8.65(8.46-8.83)$ & $8.78(8.52-9.10)$ & 0.04 \\
A. actinomycetemcomitans & $2.42(0.00-3.50)$ & $2.40(0.00-307)$ & 0.67 \\
Fusobacterium spp. & $6.62(5.82-6.85)$ & $6.37(6.00-7.03)$ & 0.08 \\
Prevotella spp. & $6.20(5.65-6.65)$ & $6.42(6.07-6.80)$ & 0.02 \\
P. micra & $4.33(3.78-5.21)$ & $5.40(4.92-5.65)$ & $0.0001^{*}$ \\
P. gingivalis & $3.90(2.56-5.70)$ & $5.52(3.07-6.04)$ & 0.04 \\
T. forsythia & $6.09(5.24-6.47)$ & $6.71(5.97-6.91)$ & $0.006^{*}$ \\
T. denticola & $5.47(4.10-6.26)$ & $6.16(5.71-6.76)$ & 0.02 \\
\hline
\end{tabular}

*Statistically significant after adjustment for multiple comparisons $(P \leq 0.0063)$; Wilcoxon signed ranks test.

detected by real-time PCR; however, in practice this is usually not possible. Some of the previous studies on periodontal pathogens were in fact not clear with respect to whether the reported lower detection limit was per reaction or per sample, but it can be concluded that at least 100 DNA copies per reaction could be detected $[11,22]$. However, a detection limit of as low as 1.6 cells per reaction has been reported $[9,10]$ which is comparable to the sensitivity of q-PCR assays in this study (5 copies/reaction). The high sensitivity of q-PCR probably explains the higher detection rates of pathogens observed in studies that employed this technique compared to those found with culture techniques or DNA-DNA hybridization.

The median log counts of total bacteria as well as some of the test species/genera in this study are 1-3 log higher than those reported by most previous studies that used real-time PCR $[9,10,12,22]$. In contrast, Lyons et al. [11] reported counts as high as $10^{14}$ and $10^{12}$ for total bacteria and $P$. gingivalis, respectively, which sounds implausible. While these differences can be attributable to variations in sampling and DNA extraction efficiency, inaccuracies in preparation of standard curves probably account for the great part of them. Curves prepared using serial dilutions of bacterial cells or genomic DNA extract, as done in most previous studies, may not be as accurate as those generated using plasmids. On the other hand, quantification bias because of plasmid DNA conformation has been reported recently, especially with circular plasmids [23]. However, since values from this study are also close to those previously obtained by checkerboard DNA-DNA hybridization [24], they can be assumed to be accurate enough.

The relative abundances of $T$. denticola and $T$. forsythia in the current study are very comparable to those reported for a Japanese population using a similar quantification technique [12]. In the latter study, however, $P$. gingivalis and Prevotella intermedia were presented at exceedingly higher proportions and showed significant association with the disease which in contrast with the current findings. Using checker board DNA-DNA hybridization, much higher proportions of the red complex members have been reported in chronic periodontitis patients from USA, Brazil, Chili and Sweden [13] compared to the relative counts described here. However, this can be simply justified by the fact that proportions in the checker board technique are calculated by normalizing absolute counts of each species to total counts of the 40 probe species used rather than total bacteria as done in the current study.

Relative quantification data show that although periodontal pathogens were present at significantly higher proportions in periodontitis sites compared to healthy sites, they still constituted a minority of subgingival

Table 4 Median (interquartile range) relative counts (\% total bacteria) of the test species/genera in subgingival plaque from the healthy and periodontitis sites

\begin{tabular}{|c|c|c|c|c|}
\hline Species & Healthy sites $(n=20)$ & Periodontitis sites $(n=20)$ & Fold difference & $P$ \\
\hline A. actinomycetemcomitans & $0.001(0.000-0.020)$ & $0.001(0.000-0.005)$ & 0.07 & 0.12 \\
\hline Fusobacterium spp. & $0.789(0.238-1.205)$ & $0.701(0.250-1.029)$ & 0.81 & 0.79 \\
\hline Prevotella spp. & $0.360(0.222-0.903)$ & $0.394(0.228-0.751)$ & 1.23 & 0.50 \\
\hline P. micra & $0.009(0.003-0.022)$ & $0.031(0.014-0.081)$ & 3.39 & $0.0001^{*}$ \\
\hline P. gingivalis & $0.004(0.0001-0.0611)$ & $0.068(0.0001-0.219)$ & 1.97 & 0.156 \\
\hline T. forsythia & $0.302(0.055-0.541)$ & $0.425(0.170-1.50)$ & 0.86 & 0.08 \\
\hline T. denticola & $0.092(0.007-0.438)$ & $0.338(0.126-0.613)$ & 1.33 & $0.006^{*}$ \\
\hline
\end{tabular}

*Statistically significant after adjustment for multiple comparisons $(P \leq 0.0063)$; Wilcoxon signed ranks test. 
microbiota. This is, however, not surprising since assessment of earlier cultivation-based studies and more recent studies employing molecular techniques clearly reveals that periodontal pathogens, particularly members of the red complex, have almost always been detected at low abundance $[3,24,25]$. What has not been addressed adequately, on the other hand, is how these pathogens can cause periodontitis at such low abundance. One interesting, currently evolving view is that low abundance periodontal pathogens orchestrate periodontitis by inducing a dysbiotic "pathogenic" microbial community that in turn mediates bone destruction [26]. This is thought to result from ability of these pathogens to subvert some components of the host response rather than to act directly as proinflammatory bacteria as has been very recently demonstrated for $P$. gingivalis in vitro [27]. Accordingly, low abundant periodontal pathogens are claimed to function as keystone pathogens, a hypothesis that challenges the role of red complex members as conventional pathogens [28].

The current study did not show an association between $P$. gingivalis and periodontal destruction, which is very hard to defend against the existing overwhelming evidence. However, this can simply be a failure to detect existing association due to lack of adequate power, especially that there was a significant difference in absolute count at the 0.05 level. On the other hand, given the polymicrobial nature of periodontitis, and in view of the new keystone pathogen hypothesis, it is also plausible to propose that other members of the pathogenic team can in certain circumstances take over the role of $P$. gingivalis as a keystone pathogen. In fact, $P$. gingivalis has not always showed the strongest association with periodontitis $[3,29,30]$. In the current study, $T$. denticola and $T$. forsythia, both members of the red complex, did show significant association with periodontitis, which is consistent with the literature. However, P. micra (previously known as Peptostreptococcus micros) showed the strongest association with the disease being present at significantly higher absolute and relative counts in periodontitis sites in all study subjects. This species is a member of the orange microbial complex [6], and there is an expanding evidence on its role, along with other peptostreptococci, as a periodontal pathogen $[3,29,31,32]$.

\section{Conclusion}

Despite its presence in very low relative counts, $P$. micra showed the strongest association with periodontal destruction, which is suggestive of a potential role as keystone pathogen in place of $P$. gingivalis. However, this needs to be validated in a larger-scale study before it can be claimed to represent ethnic variations in pathogens' association with periodontitis.
Competing interests

The authors declare that they have no competing interests.

\section{Authors' contributions}

NA designed and carried out the laboratory work, performed the statistical analysis of data and wrote the manuscript. HS was responsible of the field work, collection of specimens, and data entry. AA contributed to the study design, supervision of clinical data collection, and writing of the manuscript. IG was involved in the study design, and overall supervision of the research project. All authors read and approved the final manuscript.

\section{Acknowledgements}

This study was partially funded by Al-saeed Foundation for Science and Technology. All laboratory work was conducted at the Molecular Research Laboratory, UST, Sana'a, Yemen. We would like to thank Dr. Mohammed Sultan for his help with sample collection.

\section{Author details}

'Department of Preventive Dentistry, Faculty of Dentistry, Jazan University, P.O. box: 114, Jazan, Saudi Arabia. ${ }^{2}$ Molecular Research Laboratory, Faculty of Medical Sciences, University of Science and Technology, Sana'a, Yemen. ${ }^{3}$ Department of Periodontology, Oral Pathology, Oral Medicine and Radiology; Faculty of Dentistry, University of Sana'a, Sana'a, Yemen. ${ }^{4}$ Department of Periodontology, Faculty of Dentistry, Khartoum University, Khartoum, Sudan.

Received: 12 November 2013 Accepted: 14 February 2014 Published: 18 February 2014

\section{References}

1. Darveau RP: Periodontitis: a polymicrobial disruption of host homeostasis. Nat Rev Microbiol 2010, 8(7):481-490.

2. Moore WE, Moore LV: The bacteria of periodontal diseases. Periodontol 2000 1994, 5:66-77.

3. Kumar PS, Griffen AL, Moeschberger ML, Leys EJ: Identification of candidate periodontal pathogens and beneficial species by quantitative 16S clonal analysis. J Clin Microbiol 2005, 43(8):3944-3955.

4. Paster BJ, Boches SK, Galvin JL, Ericson RE, Lau CN, Levanos VA, Sahasrabudhe A, Dewhirst FE: Bacterial diversity in human subgingival plaque. J Bacteriol 2001, 183(12):3770-3783.

5. Dewhirst FE, Chen T, Izard J, Paster BJ, Tanner AC, Yu WH, Lakshmanan A, Wade WG: The human oral microbiome. J Bacteriol 2010, 192(19):5002-5017.

6. Socransky SS, Haffajee AD, Cugini MA, Smith C, Kent RL Jr: Microbial complexes in subgingival plaque. J Clin Periodontol 1998, 25(2):134-144.

7. Dewhirst FE, Tamer MA, Ericson RE, Lau CN, Levanos VA, Boches SK, Galvin JL, Paster BJ: The diversity of periodontal spirochetes by $16 \mathrm{~S}$ rRNA analysis. Oral Microbiol Immunol 2000, 15(3):196-202.

8. Rylev M, Kilian M: Prevalence and distribution of principal periodontal pathogens worldwide. J Clin Periodontol 2008, 35(8 Suppl):346-361.

9. Mineoka T, Awano S, Rikimaru T, Kurata H, Yoshida A, Ansai T, Takehara T: Site-specific development of periodontal disease is associated with increased levels of Porphyromonas gingivalis, Treponema denticola, and Tannerella forsythia in subgingival plaque. J Periodonto/ 2008, 79(4):670-676.

10. Yoshida A, Kawada M, Suzuki N, Nakano Y, Oho T, Saito T, Yamashita Y: TaqMan real-time polymerase chain reaction assay for the correlation of Treponema denticola numbers with the severity of periodontal disease. Oral Microbiol Immunol 2004, 19(3):196-200.

11. Lyons SR, Griffen AL, Leys EJ: Quantitative real-time PCR for Porphyromonas gingivalis and total bacteria. J Clin Microbiol 2000, 38(6):2362-2365.

12. Kuboniwa M, Amano A, Kimura KR, Sekine S, Kato S, Yamamoto Y, Okahashi N, lida T, Shizukuishi S: Quantitative detection of periodontal pathogens using real-time polymerase chain reaction with TaqMan probes. Oral Microbiol Immunol 2004, 19(3):168-176.

13. Haffajee AD, Bogren A, Hasturk H, Feres M, Lopez NJ, Socransky SS: Subgingival microbiota of chronic periodontitis subjects from different geographic locations. J Clin Periodontol 2004, 31(11):996-1002.

14. World Health Organization: Oral health surveys: basic methods. 4th edition. Geneva: World Health Organization; 1997. 
15. Silness J, Loe H: Periodontal disease in pregnancy. li. Correlation between oral hygiene and periodontal condtion. Acta Odontol Scand 1964, 22:121-135

16. Holland PM, Abramson RD, Watson R, Gelfand DH: Detection of specific polymerase chain reaction product by utilizing the $5^{\prime}--3^{\prime}$ exonuclease activity of Thermus aquaticus DNA polymerase. Proc Natl Acad Sci U S A 1991, 88(16):7276-7280.

17. Al-Hebshi NN, Al-Sharabi AK, Shuga-Aldin HM, Al-Haroni M, Ghandour I: Effect of khat chewing on periodontal pathogens in subgingival biofilm from chronic periodontitis patients. J Ethnopharmacol 2010, 132(3):564-569.

18. Suzuki N, Yoshida A, Saito T, Kawada M, Nakano Y: Quantitative microbiological study of subgingival plaque by real-time PCR shows correlation between levels of Tannerella forsythensis and Fusobacterium spp. J Clin Microbiol 2004, 42(5):2255-2257.

19. Martin FE, Nadkarni MA, Jacques NA, Hunter N: Quantitative microbiological study of human carious dentine by culture and real-time PCR: association of anaerobes with histopathological changes in chronic pulpitis. J Clin Microbiol 2002, 40(5):1698-1704.

20. Al-Otaibi M, Al-Harthy M, Gustafsson A, Johansson A, Claesson R, AngmarMansson B: Subgingival plaque microbiota in Saudi Arabians after use of miswak chewing stick and toothbrush. J Clin Periodontol 2004, 31(12):1048-1053.

21. Darout IA, Skaug N, Albandar JM: Subgingival microbiota levels and their associations with periodontal status at the sampled sites in an adult Sudanese population using miswak or toothbrush regularly. Acta Odontol Scand 2003, 61(2):115-122.

22. Nonnenmacher C, Dalpke A, Mutters R, Heeg K: Quantitative detection of periodontopathogens by real-time PCR. J Microbiol Methods 2004, 59(1):117-125

23. Lin $C H$, Chen YC, Pan TM: Quantification bias caused by plasmid DNA conformation in quantitative real-time PCR assay. PLoS One 2011, 6(12):e29101.

24. Ximenez-Fyvie LA, Haffajee AD, Socransky SS: Microbial composition of supra- and subgingival plaque in subjects with adult periodontitis. J Clin Periodontol 2000, 27(10):722-732.

25. Moore WE, Holdeman LV, Smibert RM, Hash DE, Burmeister JA, Ranney RR: Bacteriology of severe periodontitis in young adult humans. Infect Immun 1982, 38(3):1137-1148.

26. Hajishengallis G, Lamont RJ: Beyond the red complex and into more complexity: the polymicrobial synergy and dysbiosis (PSD) model of periodontal disease etiology. Mol Oral Microbiol 2012, 27(6):409-419.

27. Hajishengallis G, Liang S, Payne MA, Hashim A, Jotwani R, Eskan MA, McIntosh ML, Alsam A, Kirkwood KL, Lambris JD, et al: Low-abundance biofilm species orchestrates inflammatory periodontal disease through the commensal microbiota and complement. Cell Host Microbe 2011, 10(5):497-506

28. Hajishengallis G, Darveau RP, Curtis MA: The keystone-pathogen hypothesis. Nat Rev Microbio/ 2012, 10(10):717-725.

29. Colombo AP, Boches SK, Cotton SL, Goodson JM, Kent R, Haffajee AD, Socransky SS, Hasturk H, Van Dyke TE, Dewhirst F, et al: Comparisons of subgingival microbial profiles of refractory periodontitis, severe periodontitis, and periodontal health using the human oral microbe identification microarray. J Periodontol 2009, 80(9):1421-1432.

30. Kumar PS, Leys EJ, Bryk JM, Martinez FJ, Moeschberger ML, Griffen AL: Changes in periodontal health status are associated with bacterial community shifts as assessed by quantitative $16 \mathrm{~S}$ cloning and sequencing. J Clin Microbiol 2006, 44(10):3665-3673.

31. Rams TE, Feik D, Listgarten MA, Slots J: Peptostreptococcus micros in human periodontitis. Oral Microbiol Immunol 1992, 7(1):1-6.

32. Belstrom D, Fiehn NE, Nielsen CH, Kirkby N, Twetman S, Klepac-Ceraj V, Paster BJ, Holmstrup P: Differences in bacterial saliva profile between periodontitis patients and a control cohort. J Clin Periodontol 2014 41(2):104-112.

doi:10.1186/1472-6831-14-13

Cite this article as: Al-hebshi et al:: Subgingival periodontal pathogens associated with chronic periodontitis in Yemenis. BMC Oral Health 2014 14:13.

\section{Submit your next manuscript to BioMed Central and take full advantage of:}

- Convenient online submission

- Thorough peer review

- No space constraints or color figure charges

- Immediate publication on acceptance

- Inclusion in PubMed, CAS, Scopus and Google Scholar

- Research which is freely available for redistribution 